\title{
OPEN Identification of mesenchymal stromal cell survival responses to antimicrobial silver ion concentrations released from orthopaedic implants
}

\author{
Paul Souter ${ }^{1}$, John Vaughan ${ }^{1}$, Kerry Butcher ${ }^{1}$, Adam Dowle ${ }^{2}$, Jim Cunningham ${ }^{1}$,
} James Dodd ${ }^{1}$, Michael Hall ${ }^{1}$, Darren Wilson ${ }^{1}$, Alan Horner ${ }^{1} \&$ Paul Genever $^{2 \bowtie}$

Antimicrobial silver $\left(\mathrm{Ag}^{+}\right)$coatings on orthopaedic implants may reduce infection rates, but should not be to the detriment of regenerative cell populations, primarily mesenchymal stem/stromal cells (MSCs). We determined intramedullary silver release profiles in vivo, which were used to test relevant $\mathrm{Ag}^{+}$concentrations on MSC function in vitro. We measured a rapid elution of $\mathrm{Ag}^{+}$from intramedullary pins in a rat femoral implantation model, delivering a maximum potential concentration of $7.8 \mu \mathrm{M}$, which was below toxic levels determined for $\mathrm{MSCs}$ in vitro $\left(\mathrm{EC}_{50}, 33 \mu \mathrm{M}\right)$. Additionally, we present in vitro data of the reduced colonisation of implants by Staphylococcus aureus. MSCs exposed to $\mathrm{Ag}^{+}$prior to/during osteogenic differentiation were not statistically affected. Notably, at clonal density, the colony-forming capacity of MSCs was significantly reduced in the presence of $10 \mu \mathrm{M}$ $\mathrm{Ag}^{+}$, suggesting that a subpopulation of clonal MSCs was sensitive to $\mathrm{Ag}^{+}$exposure. At a molecular level, surviving colony-forming MSCs treated with $\mathrm{Ag}^{+}$demonstrated a significant upregulation of components of the peroxiredoxin/thioredoxin pathway and processes involved in glutathione metabolism compared to untreated controls. Inhibition of glutathione synthesis using L-buthionine sulfoxamine eliminated MSC clonogenicity in the presence of $\mathrm{Ag}^{+}$, which was rescued by exogenous glutathione.

Surgical site infection following orthopaedic trauma imparts a significant clinical and economic burden on patients and healthcare systems. It is estimated to double the median healthcare costs and patient hospitalisation time at centres within the United States ${ }^{1}$. In a European study specifically investigating infection of tibial fractures, treatment costs were estimated to increase by 6.5 -times compared to patients with no complications ${ }^{2}$.

Persistent infections are primarily caused by the presence of bacterial biofilms attached to the surfaces of implanted materials, with colonisation of fracture fixation devices caused by Staphylococcus aureus in approximately $30 \%$ of cases ${ }^{3}$. These micro-colonies are highly organised, sessile communities surrounded by extracellular polymeric substances that are able to evade both the host's defence mechanisms and antibiotics ${ }^{4-9}$. In vivo, the formation of a biofilm is greatly enhanced by the implantation of an inert material, and was the cause of failure observed in many patients ${ }^{10,11}$. It is therefore evident that the prevention of bacterial adhesion and subsequent biofilm formation can prevent implant-associated infection. With this in mind, gentamicin-coated intramedullary (IM) nails for use in fracture repair have been commercialised to block implant associated infection by Gram-negative bacteria ${ }^{12}$.

However, with the rise in antibiotic resistance, the use of a broad spectrum antimicrobial may be more appropriate. As a result, there has been growing interest in the use of silver for orthopaedic applications. In addition to the direct action on many microbial strains, the use of silver has also been shown to potentiate the effectiveness and broaden the bacterial species activity of antibiotics ${ }^{13-15}$. The wide bactericidal effects of silver are caused by disruption of the cell membrane, damage to DNA and prevention of replication, denaturation of proteins and enzymes that contribute to the loss of cell integrity as well as the disruption of energy metabolism, in part caused by the generation of reactive oxygen species (ROS) ${ }^{16-20}$. Currently, the application of silver to orthopaedic 
a

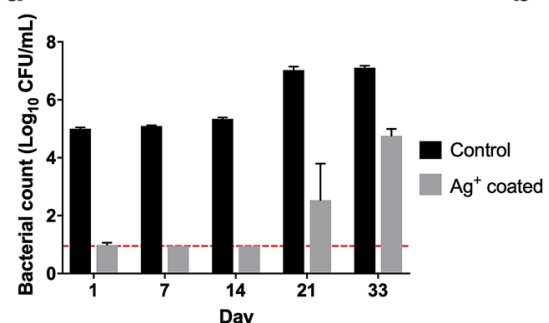

d

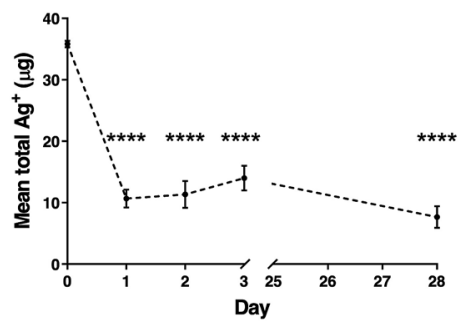

b

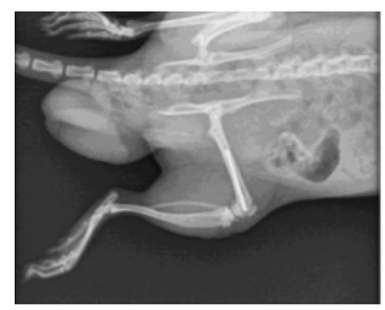

e

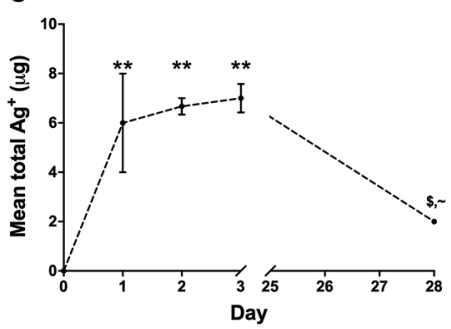

C

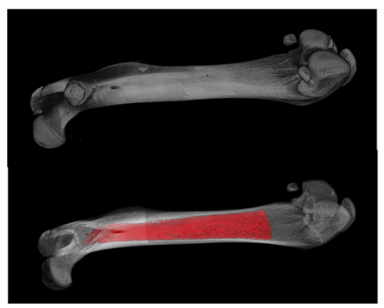

f

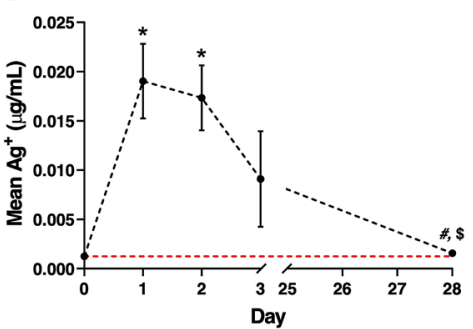

Figure 1. (a-f) Surface recovery of $S$. aureus from silver-coated grit blasted Ti64 pins following in vitro challenge (a), line represents the limit of detection of $0.95 \mathrm{CFU} / \mathrm{mL},(\mathbf{b})$ confirmation of pin implantation during in vivo assessment of silver elution, (c) microCT scan of rat femur highlighting intramedullary canal volume. Measurement of total Ag from (d) recovered implants $(\mu \mathrm{g})$, (e) operated femurs $(\mu \mathrm{g})$, and silver concentration in (f) plasma $(\mu \mathrm{g} / \mathrm{mL})$, red line represents limit of detection $(=0.00125 \mu \mathrm{g} / \mathrm{mL})$. All values are means \pm SEM $(\mathrm{n}=3)$ determined via ICP-MS for samples taken at termination (Day 1, 2, 3 and 28). Statistical analysis by one-way ANOVA using Tukey correction for multiple comparisons, significance indicated against Day 0 by ${ }^{\star} p<0.05,{ }^{* *} p$ $<0.01,{ }^{* * *} p<0.0001$, Day 1 by ${ }^{\sharp} p<0.05$, Day 2 by ${ }^{\$} p<0.05$ and Day 3 by $\sim p<0.05$.

megaprostheses implanted during bone resection as a result of osteosarcoma has proven to reduce the incidence of infection in these compromised patients ${ }^{21-23}$. The application of silver to orthopaedic trauma devices such as intramedullary nails, could therefore have a similar clinical impact.

Fracture repair requires the action of immune cells, chondrocytes and osteoblasts, the numbers and regulation of which are influenced by mesenchymal stem/stromal cells (MSCs) that act as a source or progenitors, giving rise to reparative osteochondral tissue ${ }^{24}$. MSCs are supplied to the fracture site from the surrounding soft tissue, periosteum and bone marrow, all or which may be disrupted in a severe fracture, therefore preservation of existing MSC populations and their function is of importance ${ }^{25}$. Specifically, the impact of silver ions $\left(\mathrm{Ag}^{+}\right)$ on repair of the fracture site and the subsequent generation of ROS that also occurs in eukaryotic cells, needs to be considered, particularly since the level of ROS can affect the lineage divergence of MSCs, with decreased osteogenesis occurring under in vitro conditions of oxidative stress ${ }^{26-28}$.

Here, we determined the $\mathrm{Ag}^{+}$release profile from intramedullary implants to identify how exposure to relevant in vivo concentrations of silver ions affected MSC function and survival responses in vitro. Our findings provide insight into the potential clinical use of this metal for biofilm prevention in orthopaedic trauma situations.

\section{Results}

Synthesis and characterisation of intramedullary pin implants. Silver ions were integrated on to implant surfaces and inductively coupled plasma mass spectrometry (ICP-MS) quantification of total $\mathrm{Ag}^{+}$confirmed nominal target loading of approximately $50 \mu \mathrm{g} / \mathrm{cm}^{2}$ (pins: mean, $50.36 \mu \mathrm{g} / \mathrm{cm}^{2} \mathrm{SEM} \pm 0.76, \mathrm{n}=6$ ).

Staphylococcus aureus adhesion to silver coated Ti64 grit blasted pins. Control pin implants, without $\mathrm{Ag}^{+}$coatings, supported bacterial colonisation over the test period, with a mean surface count of 5.00 $\log _{10} \mathrm{CFU} /$ sample after 1 day rising to $7.11 \log _{10} \mathrm{CFU} /$ sample at day 33 (Fig. 1a). Colonisation was significantly reduced on $\mathrm{Ag}^{+}$coated pins ( $>4 \log _{10} \mathrm{CFU} /$ sample) over control counts at $24 \mathrm{~h}$, maintaining this distinction at day 7 and 14 re-challenges ( $>4 \log _{10} \mathrm{CFU} /$ sample). At these time points all replicates displayed counts at or below the detection limit. At day $21, \mathrm{Ag}^{+}$coated samples displayed a degree of colonisation, however values remained below control counts by $>4 \log _{10} \mathrm{CFU} /$ sample. Colonisation was detected in $\mathrm{Ag}^{+}$coated pins at day 33 that was comparable to control samples at earlier time points (day 1, 7 and 14), but still reduced compared to control (Fig. 1a).

Rapid elution of $\mathrm{Ag}^{+}$from implants within intramedullary canal environment. To determine $\mathrm{Ag}^{+}$elution kinetics, $\mathrm{Ag}^{+}$coated titanium pins $\left(\mathrm{Ag}^{+}, 50.36 \mu \mathrm{g} / \mathrm{cm}^{2} \mathrm{SEM} \pm 0.76\right)$ were implanted in to rat femoral intramedullary canals (Fig. $1 \mathrm{~b}, \mathrm{c}$ ) and the $\mathrm{Ag}^{+}$determined in the recovered implant (Fig. 1d), the femurs receiving the implants (Fig. 1e) and plasma (Fig. 1f). Results revealed a burst release within the $24 \mathrm{~h}$ following implantation, equating to approximately $70 \%$ (mean $25.15 \mu \mathrm{g}, \mathrm{SEM} \pm 1.45 \mu \mathrm{g}$ ) of the pre-implantation content, with little significant release over the remaining study period (Fig. 1d). A corresponding rapid elevation in plasma and femoral $\mathrm{Ag}^{+}$content was detected at Day 1 that remained elevated at Day 3 (Fig. 1e,f). Maximum 

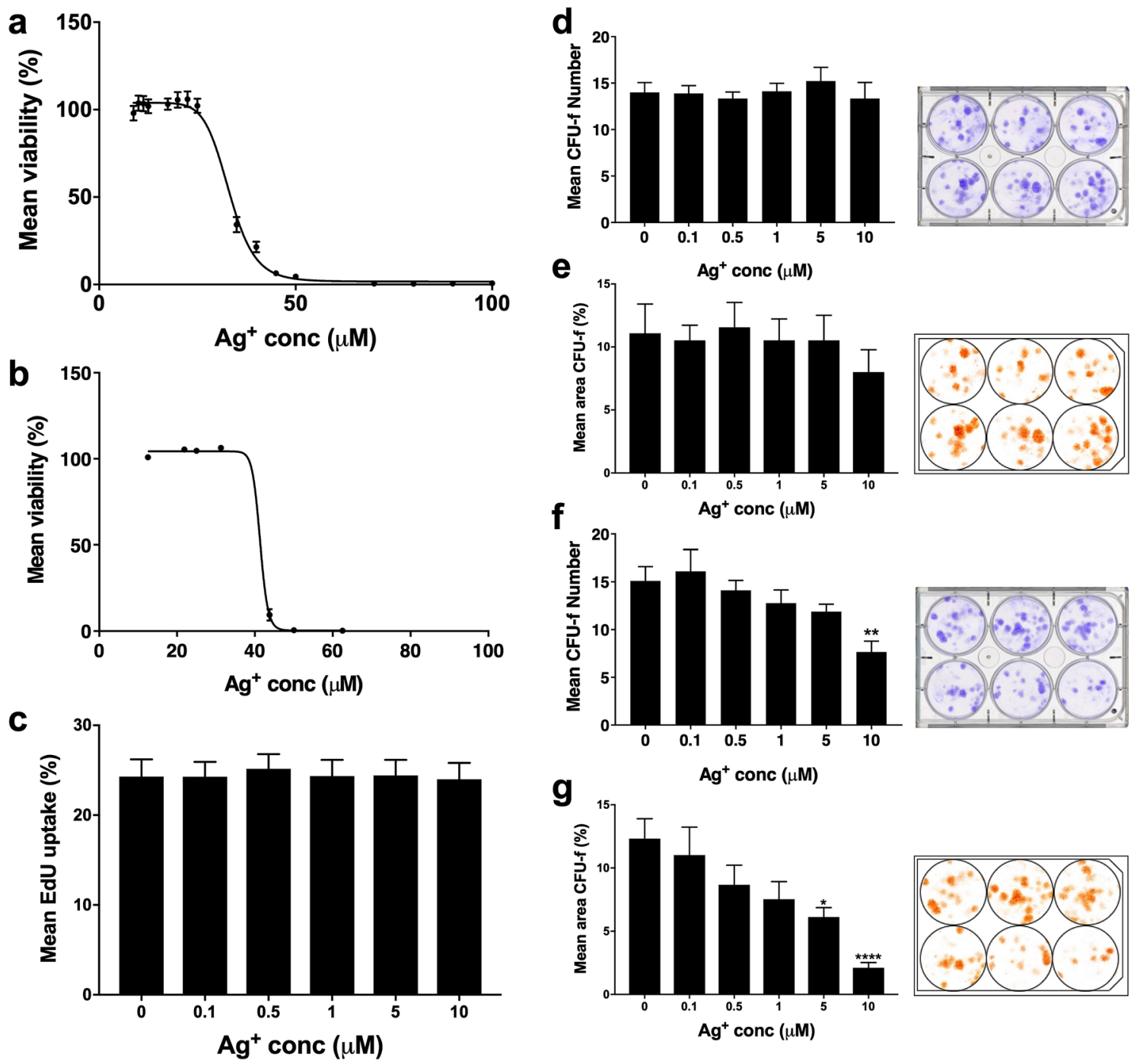

Figure 2. (a-g) Effect of $\mathrm{Ag}^{+}$dose response on percentage viability of (a) hObs $\left(\mathrm{EC}_{50}: 41.4 \mu \mathrm{M}\right)$ and (b) MSCs $\left(\mathrm{EC}_{50}: 33.06 \mu \mathrm{M}\right)$. (c) proliferation (EdU) and CFU-f number $(\mathbf{d})$ and area $(\mathbf{e})$ of MSCs at sub-EC $\mathrm{E}_{50}$ concentrations $(\leq 10 \mu \mathrm{M})$ during 3 day exposure. CFU-f number $(\mathbf{f})$ and area $(\mathbf{g})$ during continuous $\mathrm{Ag}^{+}$ exposure. All values are means \pm SEM $(n=3)$. Images in the right panel are representative of 6-well plates, stained with crystal violet (blue) and analysed (brown) for CFU-f number and area; $\mathrm{Ag}^{+}$concentrations for the plates are (L-R): top row, 0, 0.1, $0.5 \mu \mathrm{M}$; bottom row, 1, 5, $10 \mu \mathrm{M}$. Statistical analysis was performed for all $\mathrm{Ag}^{+}$groups compared to control (by one-way ANOVA using Dunnett's correction for multiple comparisons), significance indicated by ${ }^{\star} p<0.05,{ }^{* *} p<0.01,{ }^{* * *} p<0.0001$.

plasma concentration $(0.019 \mu \mathrm{g} / \mathrm{mL}, \mathrm{SEM} \pm 0.004 \mu \mathrm{g} / \mathrm{mL})$ declined following the Day 1 peak and was reduced to baseline over the remaining study period. Femoral $\mathrm{Ag}^{+}$content at Day $3(7.00 \mu \mathrm{g}$; SEM $\pm 0.58 \mu \mathrm{g})$ significantly decreased by Day $28(2 \mu \mathrm{g})$ (Fig. 1f).

The free canal volume (i.e. available canal volume with implant in situ) determined from microCT reconstructions (Fig. 1c) equated to $43 \mu \mathrm{L}$, therefore the theoretical maximum $\mathrm{Ag}^{+}$concentration within the canal following initial release equated to $6.00 \mu \mathrm{M}$ (mean $5.42 \mu \mathrm{M}$, SEM \pm 0.31 , Supplementary Table S1). In the event of instant total release of $\mathrm{Ag}^{+}$from an implant, $\mathrm{Ag}^{+}$within the canal was calculated to reach a peak of $7.97 \mu \mathrm{M}$ (mean $7.72 \mu \mathrm{M}, \mathrm{SEM} \pm 0.12$ ). For the purpose of in vitro testing, an $\mathrm{Ag}^{+}$value of $10 \mu \mathrm{M}$ was taken forwards as a maximum test concentration. Further data detailing Day 1 implant elution, total plasma $\mathrm{Ag}^{+}$and unaccounted $\mathrm{Ag}^{+}$are available in Supplementary Table S1.

In vitro susceptibility of MSCs to $\mathrm{Ag}^{+}$is increased at clonal density. The effect of $\mathrm{Ag}^{+}$dose on primary MSC and human osteoblast (hObs) cultures provided calculated $\mathrm{EC}_{50}$ values of $33.06 \mu \mathrm{M}$ and $41.4 \mu \mathrm{M}$, respectively (Fig. 2a,b). MSC proliferation in the presence of $\mathrm{Ag}^{+}$was unaffected over 3 days at concentrations below the $\mathrm{EC}_{50}$ as determined by EdU incorporation (Fig. 2c). Colony-forming capacity of MSCs as measured by colony number and percentage area was also unaffected by exposure to sub-EC $\mathrm{Eg}_{50} \mathrm{Ag}^{+}$concentrations during the initial 3 days of culture (which broadly replicated the exposure period determined during the in vivo elution 

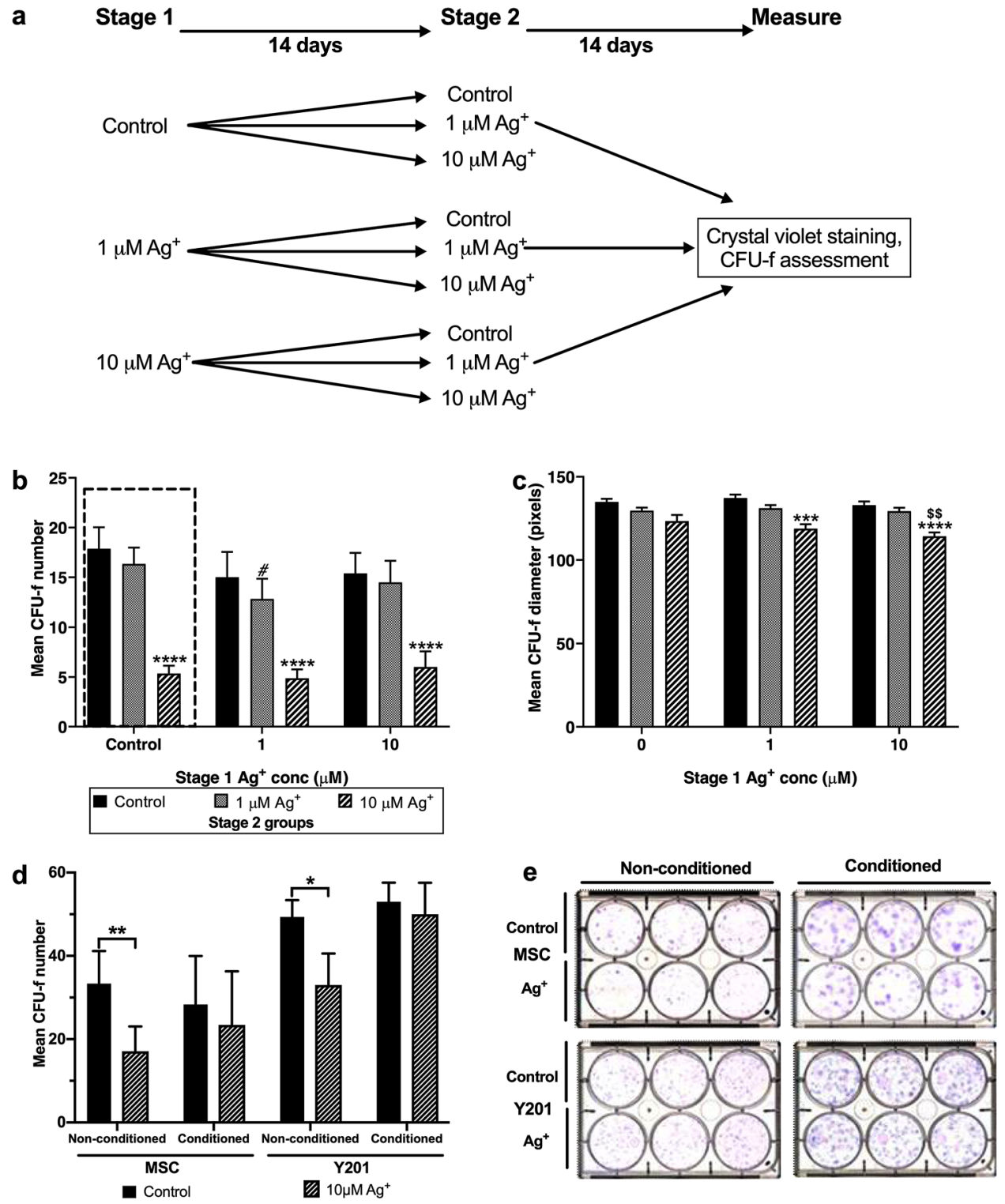

Figure 3. (a-e) Two-stage assay design (a) used to determine the continued MSC colony-forming capacity and $\mathrm{Ag}^{+}$tolerance. Clonal density MSCs were cultured for 14 days $\pm \mathrm{Ag}^{+}$after which colonies of treatment groups were pooled and reseeded at clonal density and re-cultured $\pm \mathrm{Ag}^{+}$for a further 14 days. The resulting clonogenic capacity was measured, represented by (b) CFU-f number and (c) CFU-f diameter. $\mathrm{Ag}^{+}$exposure during initial culture is represented on the $\mathrm{x}$-axis with different $\mathrm{Ag}^{+}$exposures during Stage 2 displayed. Values represent mean $( \pm S E M, n=3)$. Statistical analysis by ANOVA; both using appropriate correction for multiple comparisons. Analysis performed for data within each 'Stage 1' $\mathrm{Ag}^{+}$concentration, comparing against control, represented by ${ }^{* * *} p<0.001{ }^{* * *} p<0.0001$; and against $1 \mu \mathrm{M} \mathrm{Ag}{ }^{+}$represented by ${ }^{\$ \$} p<0.005$. Comparison of each

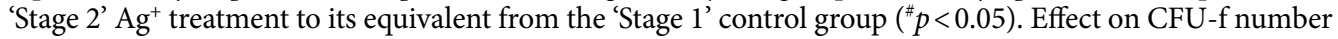
when $\mathrm{Ag}^{+}$is applied in MSC-CM. (d) Effect of $10 \mu \mathrm{M} \mathrm{Ag}^{+}$treatment on CFU-f number $( \pm$SEM, $\mathrm{n}=3)$ in the presence of non-conditioned and MSC-conditioned medium using primary MSCs $(p=0.52)$ and Y201 MSCs $(p=0.81)$ in the CFU-f assays. Statistical analysis by two-way ANOVA using Sidak's correction for multiple comparisons, significance indicated by ${ }^{\star} p<0.05,{ }^{*} p<0.001$. (e) Representative images of crystal violet stained CFU-f for primary MSCs and Y201 MSCs.

study) (Fig. 2d,e). However, when MSCs were treated with $\mathrm{Ag}^{+}$for the duration of the assay period, $10 \mu \mathrm{M} \mathrm{Ag}^{+}$ caused a significant reduction in colony number and area (Fig. 2f,g).

MSC colony formation is unaffected by previous exposure to $\mathrm{Ag}^{+}$. Maintenance of MSC clonal expansion capacity following $\mathrm{Ag}^{+}$exposure is necessary for the continued functioning of bone marrow stroma during and subsequent to fracture repair. This property was determined through the secondary seeding of $\mathrm{Ag}^{+}$ exposed CFU-f using a two-stage assay design (Fig. 3a). Essentially, CFU-f were exposed to $\mathrm{Ag}^{+}$at 0,1 and $10 \mu \mathrm{M}$ 
concentrations for 14 days (Stage 1). After this time, the surviving CFU-fs from each treatment were trypsinised, re-plated at CFU-f density in fresh plates and re-exposed to $\mathrm{Ag}^{+}$at 0,1 and $10 \mu \mathrm{M}$ concentrations for a further 14 days (Stage 2). This protocol enabled us to test the hypothesis that colonies surviving an initial $\mathrm{Ag}^{+}$exposure represented an $\mathrm{Ag}^{+}$resistant MSC subpopulation. Stage $2 \mathrm{CFU}-\mathrm{f}$ number for untreated controls was unaffected by previous exposure to $\mathrm{Ag}^{+}$. However, the surviving Stage $1 \mathrm{Ag}^{+}$exposed MSCs remained susceptible to further $\mathrm{Ag}^{+}$exposure in Stage 2 with significant decreases in CFU-f number, irrespective of initial $\mathrm{Ag}^{+}$treatment (Fig. 3b) indicating that $\mathrm{Ag}^{+}$exposure did not select for a resistant MSC subtype.

An inverse correlation was observed between CFU-f diameter and $\mathrm{Ag}^{+}$concentration (Fig. 3c). This relationship was apparent irrespective of pre-exposure to $\mathrm{Ag}^{+}$, however the reduction was only significant for those colonies derived from MSCs previously cultured with silver.

Conditioned medium from MSCs maintains clonogenicity during exposure to $\mathrm{Ag}^{+}$. We have found that colony-forming MSCs grown at single cell densities are much more sensitive to $\mathrm{Ag}^{+}$exposure than confluent or near confluent MSC cultures. We hypothesised that this may be due to the decreased availability of secreted, protective signalling factors in CFU-f cultures compared to more densely packed MSC monolayers. To test this hypothesis, conditioned medium (CM) was collected from confluent MSC cultures and applied to the CFU-f assays in the presence and absence of silver ions. We found that the CFU-f number reduction observed at $10 \mu \mathrm{M} \mathrm{Ag}{ }^{+}$could be rescued by the addition of CM (Fig. 3d). Both human primary MSCs and the Y201 human MSC line (used here as a reproducible model human MSC clonal line ${ }^{29}$ ) showed recovery of CFU-f number near to that of control when treated with $\mathrm{Ag}^{+}$in $\mathrm{CM}$. There was also a notable increase in colony size of the CM groups compared to unconditioned medium controls (Fig. 3e).

Glutathione synthesis is upregulated in MSCs of surviving CFU-f in response to $\mathrm{Ag}^{+}$. We have shown that a proportion of MSCs persist as CFU-fs following exposure to $\mathrm{Ag}^{+}$ions, suggesting that $\mathrm{Ag}^{+}$may activate survival mechanisms in MSCs, depending on cell density. To identify these mechanisms, Y201 MSCs were used in a proteomic screen of $\mathrm{Ag}^{+}$exposed CFU-f compared to untreated control CFU-f. Liquid chromatography tandem mass spectrometry (LC-MS/MS) proteomic analysis identified 5050 quantifiable proteins, with peak area-based label-free relative quantification applied using Progenesis QI software (Fig. 4a). Of those identified, 89 were significantly upregulated in $\mathrm{Ag}^{+}$exposed CFU-f compared to controls, while 21 were significantly downregulated (FDR <0.05) (Fig. 4b). Supplementary figures S1(a-d) demonstrate the differentiation of sample groups and consistency of biological replication in the proteomic data set. Gene Ontology (GO) analysis identified several differentially regulated functional responses including several metabolic pathways, as well as cellular detoxification (GO:1990748), response to stress (GO:0006950) and response to xenobiotic stimulus (GO:0009410) (Fig. 4c). Comparison of proteomic data to known KEGG pathways highlighted glutathione metabolism as the major upregulated cellular response to $\mathrm{Ag}^{+}$exposure. Further KEGG pathway analysis drew attention to the upregulation of proteins involved with DNA replication and nucleotide excision repair (PCNA, LIG1, CUL4A).

Validation of the proteomic evidence was provided by quantitative Polymerase Chain Reaction (qPCR) analysis of the oxidative stress response of three primary MSC donors (Fig. 4d). Data were normalised to B2M, which identified 39 genes showing mean fold-change $>1.5$ (Fig. 4e). Genes of glutathione synthesis/metabolism (GPX3, GPX5, GPX6, GR, GCLM), peroxiredoxin (PRDX1) and thioredoxin (TXN, TXNRD1) showed activation of these pathways upon $\mathrm{Ag}^{+}$exposure. Additionally, increases in the stress inducible regulator of NRF2, sequestome 1 (SQSTM1), heme oxygenase-1 (HMOX1), NAD(P)H:quinone oxidoreductase-1 (NQO1) and SOD1 were also noted for all donors. GO functional analysis related to biological processes was performed on the qPCR data, with an FDR (false discovery rate) below 5\% the threshold for significance (Supplementary Fig. S2). Highlights of those terms include: the removal of superoxide radicals (GO:0019430), response to hydrogen peroxide (GO:0042542), regulation of reactive oxygen species metabolic process (GO:2000377) and glutathione metabolic process (GO:0006749). Furthermore, cellular response to cadmium (GO:0071276) and cellular copper homeostasis (GO:0006878) all indicate at the mechanisms employed to ensure management of metal ions that could result in toxicity. Of those identified, glutathione metabolic process (GO:0006749) was replicated from the proteomic analysis (Supplementary Fig. S3), the only functional process to be so.

Using primary MSCs, we identified increased GCLM immunostaining, a component of glutathione synthesis, and thioredoxin in $\mathrm{Ag}^{+}$exposed CFU-f compared to untreated controls (Fig. 4f). Analysis of whole colony area did not reveal any variation in the expression of either protein.

We used a pharmacological inhibitor of glutathione synthesis, L-buthionine sulfoxamine (BSO), to determine the functional effects of glutathione on CFU-f survival while under $\mathrm{Ag}^{+}$exposure conditions. As observed before, a significant reduction in CFU-f number was noted at $10 \mu \mathrm{M} \mathrm{Ag}^{+}$in the absence of glutathione inhibition. However with the addition of $1 \mu \mathrm{M}$ BSO, a significant reduction in CFU-f was observed with $\mathrm{Ag}^{+} \geq 5 \mu \mathrm{M}$, compared to their equivalent $\mathrm{Ag}^{+}$only control (Fig. $4 \mathrm{~g}$ ). In the case of $10 \mu \mathrm{M} \mathrm{Ag}^{+}$, CFU-f formation was eliminated in the presence of BSO. Recovery of CFU-f number was achieved through the concomitant treatment of $10 \mu \mathrm{M} \mathrm{Ag}^{+}$/ BSO with exogenous glutathione (GSH-MEE).

Effects of silver on adipogenic differentiation is dependent on exposure time. The level of lipid deposition by MSCs as measured by Oil Red O staining revealed a reduction in adipogenesis when exposed to silver compared to controls. While a short term exposure during the initial 3 days of differentiation had no effect, continuous silver treatment during differentiation resulted in significant declines compared to untreated control and short term sliver exposure $(p<0.001)$ (Supplementary Fig. S4a). In contrast, pre-treatment of MSCs 
a
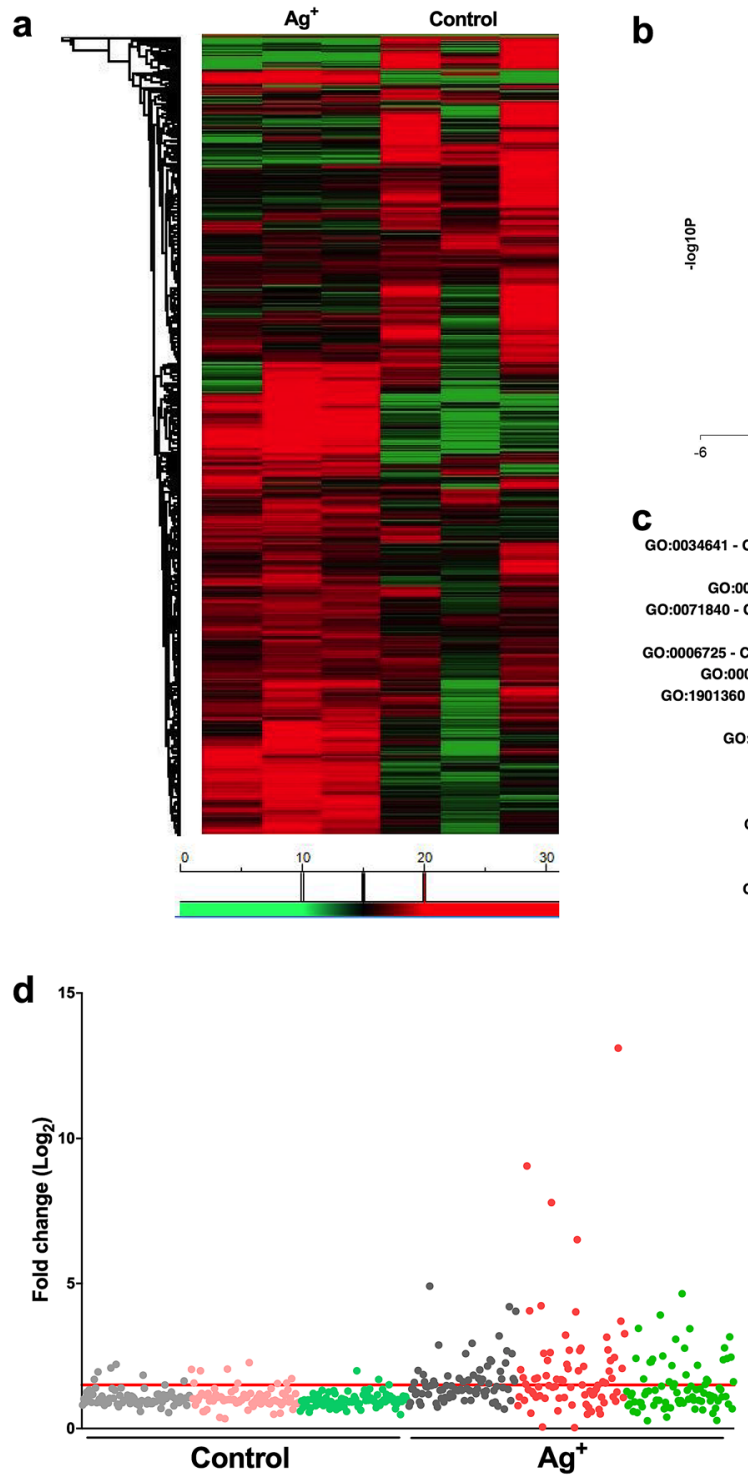

e

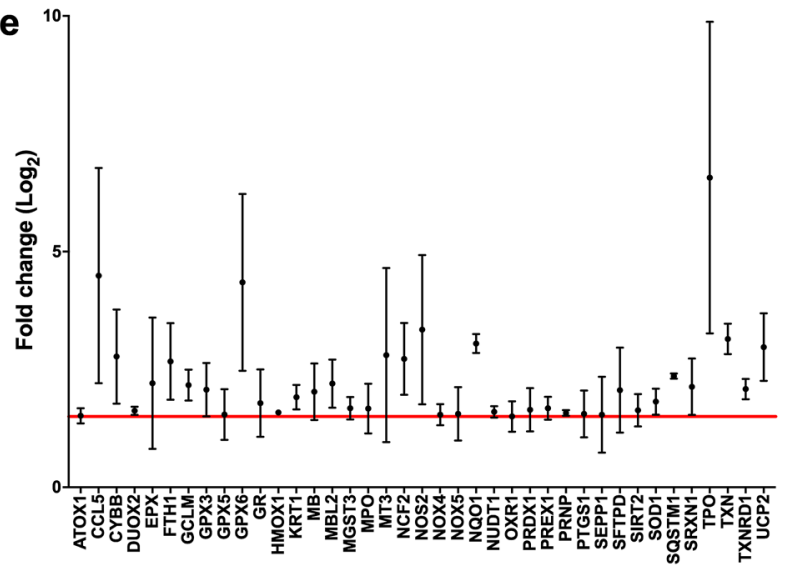

b

C
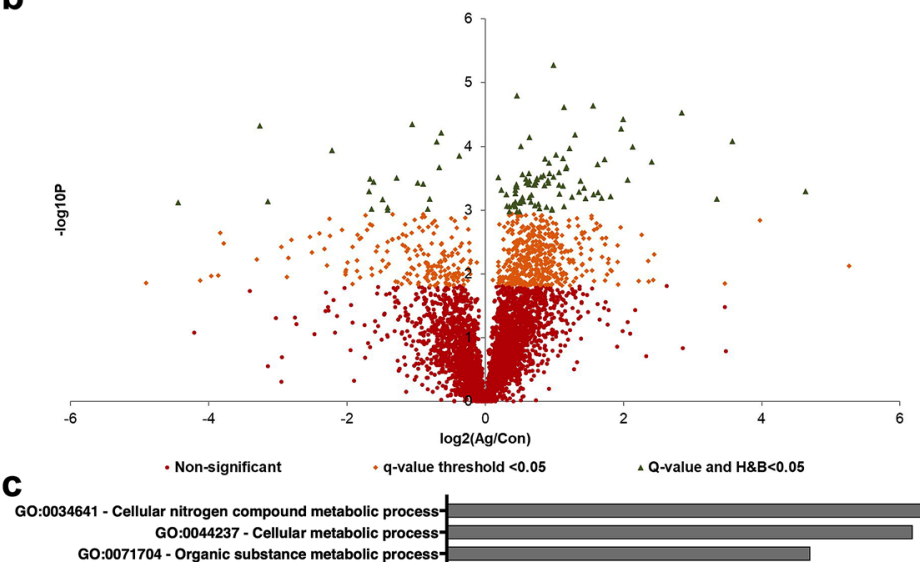

- Cellular metabolic process

GO:0071840 - Cellular component organization or biogenesi

GO:0008152 - Metabolic proces

GO:0006725 - Cellular aromatic compound metabolic process -

GO:0006807 - Nitrogen compound metabolic process

O:1901360 - Organic cyclic compound metabolic proces

G0:1990748 - Cellular detoxification-

GO:0070727 - Cellular macromolecule localization -

GO:0034613 - Cellular protein localization-

0:0006749 - Glutathione metabolic proces

GO:0033554 - Cellular response to stress-

GO:0016043 - Cellular component organization-

GO:0006950 - Response to stress-

GO:0009314 - Response to radiatio

GO:0009410 - Response to xenobiotic stimulus-

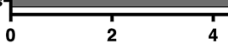

$\begin{array}{lr}4 & 6 \\ \text { FDR (-Log2) }\end{array}$

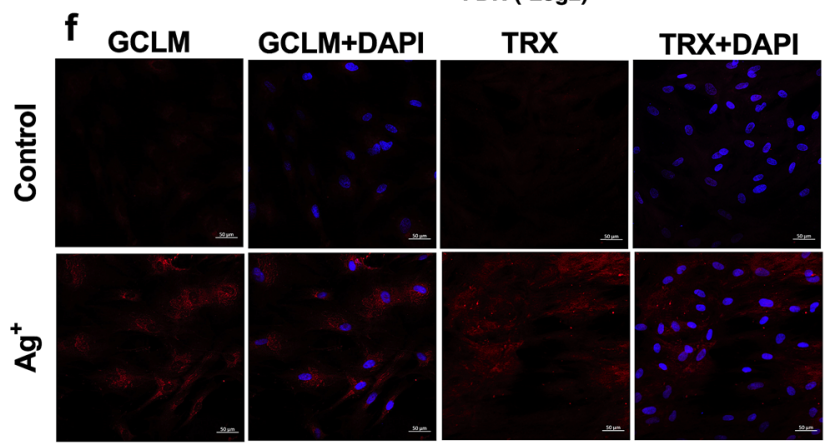

g

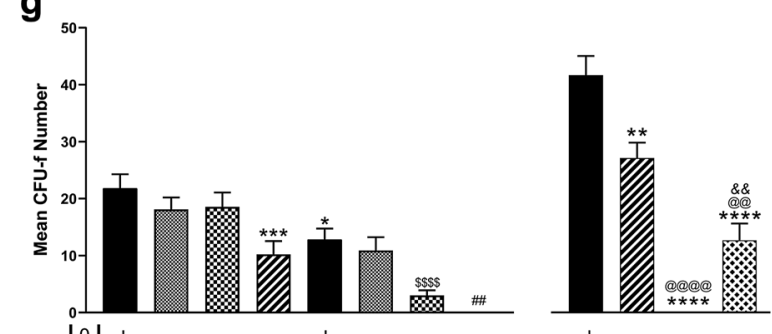


4Figure 4. (a-g) Proteomic and molecular identification of the oxidative stress response of MSCs during exposure to $\mathrm{Ag}^{+}$. (a) Protein relative abundance heat map from Y201 CFU-f under control and $\mathrm{Ag}^{+}$exposure conditions (green $<15$, red $>20$ ). (b) Volcano plot of 5050 quantified proteins, negative/positive $\log _{2}$ data equate to $\mathrm{Ag}^{+}$group down/upregulated, respectively. $15.19 \%$ of proteins were significantly differentially regulated accepting Progenesis QI calculated q-value of $<0.05$ (orange), reducing to $2.17 \%$ (green) when applying additional multiple test correction to 5\% FDR (Benjamini and Hochberg). (c) GO terminology of biological processes (FDR $<0.05$ ) associated with differentially upregulated proteins of $\mathrm{Ag}^{+}$cultured Y201 CFU-f. (d) Fold change of oxidative stress response genes in 3 primary MSC donors (grey, pink/red and green; dots represent different genes), the red line represents 1.5 fold-change. (e) Oxidative stress genes exhibiting $\geq 1.5$ fold-change following $\mathrm{Ag}^{+}$exposure versus controls, values are mean $\pm \operatorname{SEM}(\mathrm{n}=3)$. (f) Immunostaining of CFU-f for GCLM and TRX (both red), counterstained with DAPI (blue). Scale bar: $50 \mu \mathrm{m}$. (g) $\mathrm{Ag}^{+}$induced reduction of CFU- $\mathrm{f}$ is potentiated by inhibition of glutathione synthesis with $\mathrm{BSO}$, with recovery aided by exogenous GSH. Results represent the mean CFU-f number \pm SEM $(n=3)$. Statistical analysis by one-way ANOVA using Sidak's correction for multiple comparisons. Comparisons performed against control represented by ${ }^{\star} p<0.05$, ${ }^{* *} p<0.01,{ }^{* * *} p<0.001,{ }^{* * *} p<0.0001$. Additional comparisons made against treatment groups containing the same level of $\mathrm{Ag}^{+}$(e.g. $1 \mu \mathrm{M} \mathrm{Ag}^{+} \mathrm{v} 1 \mu \mathrm{M} \mathrm{Ag}^{+} / 1 \mu \mathrm{MBSO}$ ), significance indicated compared to $5 \mu \mathrm{M} \mathrm{Ag}^{+}(\$)$ and $10 \mu \mathrm{M} \mathrm{Ag}^{+}(\#)$. Exogenous GSH in the presence/absence of $10 \mu \mathrm{M} \mathrm{Ag}+$ and $1 \mu \mathrm{M}$ BSO. Comparisons performed against control as before, with additional comparisons made against $\mathrm{Ag}^{+}(@)$ and $\mathrm{Ag}^{+} / \mathrm{BSO}(\&)$.

with silver during expansion, with subsequent silver-free differentiation, caused a slight but significant increase in adipogenesis compared to control ( $p<0.05$, Supplementary Fig. S4b).

Osteogenic differentiation of MSCs is unaffected by $\mathrm{Ag}^{+}$exposure. The effect of $\mathrm{Ag}^{+}$exposure on MSC osteogenic differentiation capacity was assessed by measurement of alkaline phosphatase (ALP) activity. Osteogenic differentiation of MSCs was performed in the presence and absence of $10 \mu \mathrm{M} \mathrm{Ag} \mathrm{g}^{+}$, with ALP activity normalised to DNA determined for short-term (3 day) exposure and continual $\mathrm{Ag}^{+}$exposure groups (Fig. 5a). Cultures treated during the initial 3-day period exhibited a $16 \%$ decline from control, while a $26 \%$ reduction was observed for cultures treated for the entire differentiation period, however both findings did not reach significance ( $p=0.582$ and $p=0.246$, respectively) (Fig. 5a and a,i). Furthermore, analysis of DNA data provided additional evidence of the long-term viability of confluent cultures at continual sub-EC $\mathrm{E}_{50} \mathrm{Ag}^{+}$concentrations (Fig. 5a, ii).

We had previously found that $\mathrm{Ag}^{+}$exposure could inhibit MSC CFU-f capacity. We therefore determined if the osteogenic differentiation of MSCs was affected following CFU-f culture in the presence of $\mathrm{Ag}^{+}$. We found that osteogenic capacity (as measured by normalised and total ALP activity) was unaffected by previous exposure to $\mathrm{Ag}^{+}$during CFU-f (Fig. 5b and b,i). However, and in contrast to previous observations, DNA counts (relating to cell number) were significantly elevated in cultures from MSCs previously exposed to $\mathrm{Ag}^{+}$(Fig. 5b,ii).

CFU-Ob capacity is inhibited during $\mathrm{Ag}^{+}$exposure. MSCs were plated at colony-forming seeding density and then exposed to osteogenic conditions to determine the effect of $\mathrm{Ag}^{+}$exposure on the formation of CFU-Ob. In a manner similar to the effects of $\mathrm{Ag}^{+}$exposure on CFU-f growth, the generation of ALP-positive $\mathrm{CFU}-\mathrm{Ob}$ was decreased in the presence of continuous $\mathrm{Ag}^{+}$. Colony number in the $10 \mu \mathrm{M} \mathrm{Ag}{ }^{+}$treatment group declined by $62 \%$ from control but did not reach statistical significance ( $p=0.112$, Fig. $5 c)$, however total CFU-Ob area was significantly reduced at this sub-EC ${ }_{50}$ concentration (Fig. $5 \mathrm{~d}$ ).

\section{Discussion}

The consequences of infection of orthopaedic trauma devices are both severe for the patient and costly to the healthcare system ${ }^{2,30}$. Although the risk of infection for open tibial fractures is as high as $36 \%$, the primary aim of treating all at risk injuries with implants comprising an anti-microbial technology should not jeopardise successful union ${ }^{31}$. Here, we have investigated the use of silver as a broad spectrum antimicrobial in the prevention of biofilm formation, specifically its elution within an intramedullary setting. We then used the in vivo elution profile data to determine in vitro effects on MSC colony-forming capacity and differentiation, the mechanisms of MSC tolerance to $\mathrm{Ag}^{+}$induced oxidative stress, and the outcomes of fracture healing.

The elution profile of any additive from an implant is of importance for not only efficacy but also determining the safety of such products during pre-clinical evaluations. With regards to testing silver-coated implants, valuable data can be gained from laboratory-based testing, for example using saline or simulated body fluid as carrier solutions. However due to the chemical complexities of using this transition metal, the analysis of silver elution from implants placed in clinically relevant in vivo localities is essential ${ }^{32,33}$. Previous reporting of release profiles from silver technologies have shown a rapid increase in silver at day 2 following sub-cutaneous implantation, declining thereafter from day $6^{15}$. Consistent with those data, we also found a rapid elevation in silver concentration at the implantation site, additionally correlating this to the concomitant rise in plasma concentrations and a large decline of implant-associated $\mathrm{Ag}^{+}$. Importantly, this was mapped during the initial period following implantation and allowed calculation of the maximal $\mathrm{Ag}^{+}$burst release within the free canal space, which was applied in our in vitro investigation. While the elution of some pharmaceuticals has been assessed in this way, to our knowledge, the determination of silver elution from such an implant location has not previously been described $^{34}$. Importantly, the same implants proved capable of reducing in vitro colonisation by $S$. aureus over a 14 day period, a pathogen commonly the cause of implant related infection ${ }^{3}$. 
a

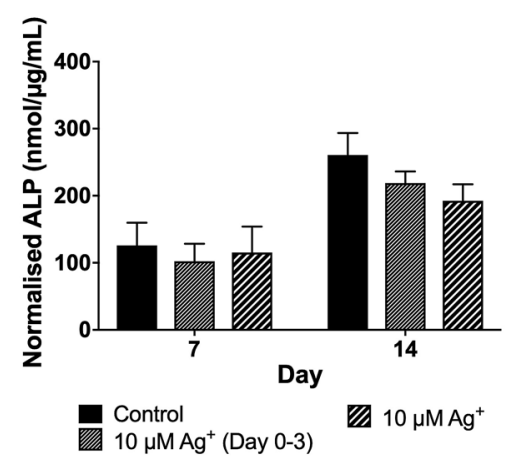

i

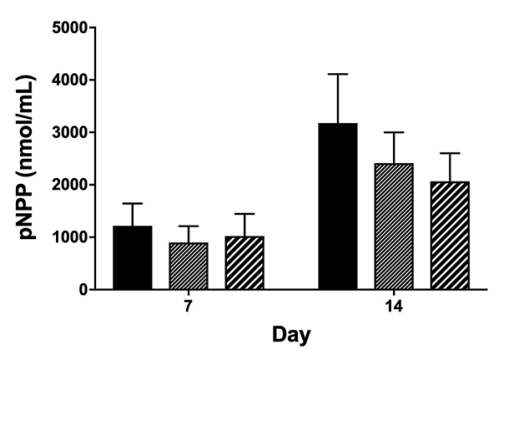

ii

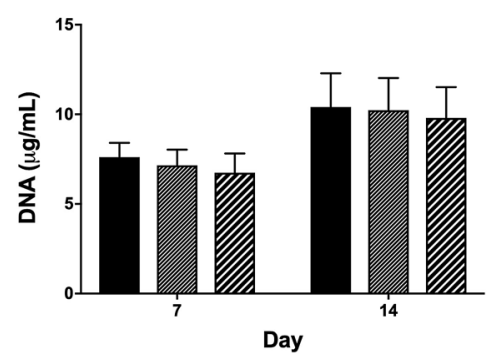

b

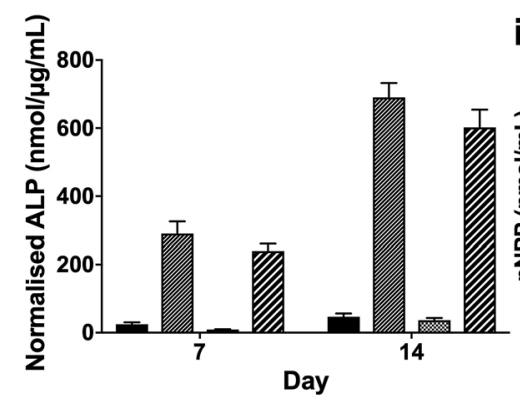

Control CFU-f - Non-osteogenic . $\mathrm{Ag}^{+} \mathrm{CFU}-\mathrm{f}$ - Non-osteogenic

שVI Control CFU-f - Osteogenic $\mathrm{Ag}^{+}$CFU-f - Osteogenic

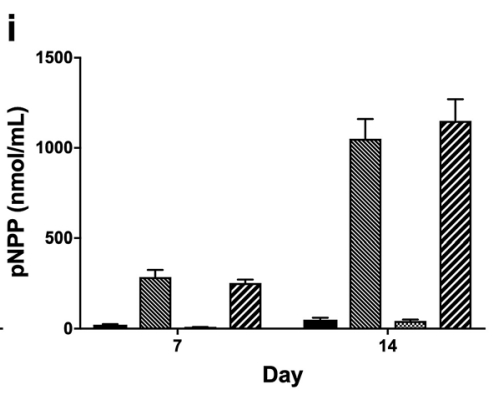

Day

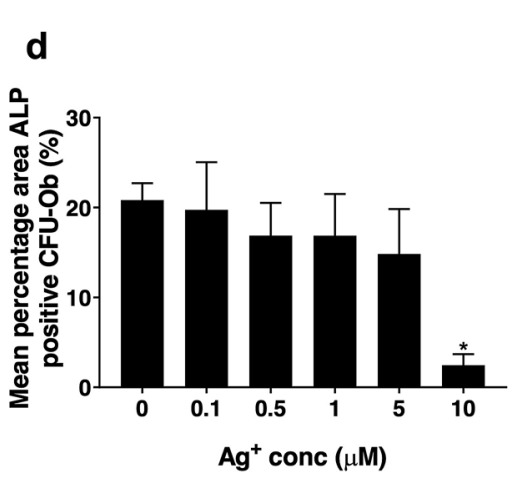

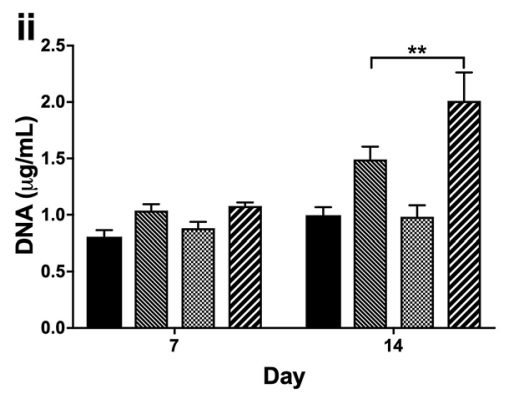

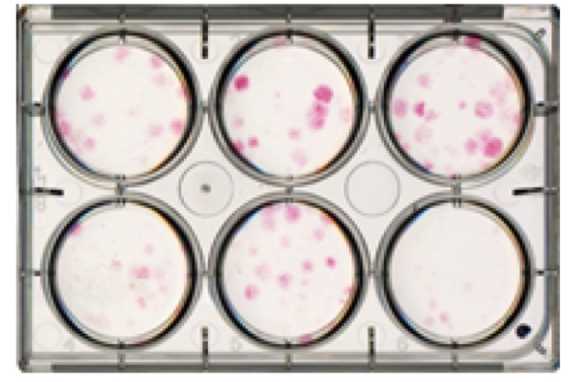

C

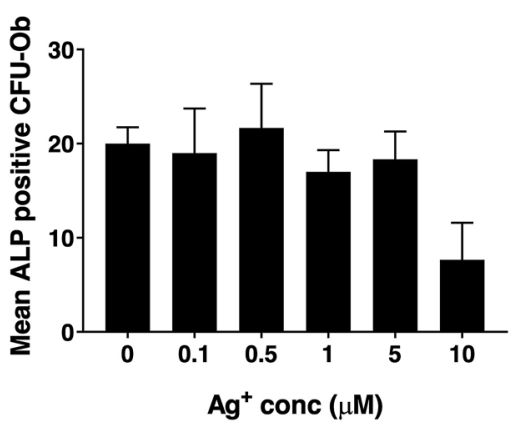

Figure 5. (a-d) Normalised ALP activity of MSCs following $\mathrm{Ag}^{+}$exposure (a) during, and (b) prior to differentiation. (a) Normalised ALP activity in the presence of $10 \mu \mathrm{M} \mathrm{Ag}^{+}$for either the initial 3 days of culture $\left(\mathrm{Ag}^{+} 10 \mu \mathrm{M}\right.$ (Day 0-3)) or for the entire differentiation period $\left(\mathrm{Ag}^{+} 10 \mu \mathrm{M}\right)$. Control cultures received $\mathrm{Ag}^{+}$free osteogenic differentiation medium only. Total ALP activity (i) and DNA quantification (ii) were measured from cell lysates. (b) Normalised ALP activity of CFU-f derived MSCs cultured in the presence/absence of $10 \mu \mathrm{M}$ $\mathrm{Ag}^{+}$prior to osteogenic differentiation in $\mathrm{Ag}^{+}$free medium. Total ALP activity (i) and DNA quantification (ii) were measured from cell lysates. The results represent the mean \pm SEM for three donors, performed in triplicate. CFU-Ob, (c) number, and (d), total percentage area during $\mathrm{Ag}^{+}$exposure. All values are means $\pm \mathrm{SEM}(\mathrm{n}=3)$. Representative image (right) of CFU-Ob, with $\mathrm{Ag}^{+}$concentrations for plates (L-R): top row, 0, 0.1, $0.5 \mu \mathrm{M}$; bottom row, 1, 5, $10 \mu \mathrm{M}$. Statistical analysis performed by ANOVA using appropriate correction for multiple comparisons. Significance against control indicated by ${ }^{\star} p<0.05,{ }^{\star *} p<0.01$.

MSCs account for $<0.01 \%$ of the cells isolated from the bone marrow, therefore the capacity to proliferate from a low density population provides the niche with a rapid source of progenitors required for skeletal remodelling and repair ${ }^{35}$. The maintenance of a viable population of colony-forming stromal cells is of paramount importance, and the CFU-f assay can be used as an in vitro assessment of this ability ${ }^{36,37}$. Through this assay we revealed a reduction of MSC colony-forming capacity in the presence of sub-toxic $\mathrm{Ag}^{+}$that prompted the analysis of the mechanisms of tolerance employed by the surviving colonies. ROS production following heavy metal ion exposure is regulated by superoxide dismutase (SOD) and catalase (CAT) in addition to several other antioxidant enzymes and their components, such as the predominant source of cellular thiol groups, glutathione ${ }^{38}$. However, variations in oxidative stress protein synthesis between tissues have been identified during in vivo cadmium exposure $^{39}$. In vitro molecular and protein profiling of these cellular mechanisms has ordinarily been performed after short term exposure using confluent cultures, while investigating specific proteins involved in oxidative 
stress management ${ }^{40-42}$. Significantly, for orthopaedic applications, the data presented here provides an insight into the global mechanisms employed by chronically exposed $\mathrm{Ag}^{+}$tolerant clonogenic MSCs.

The glutathione synthesis inhibitor, BSO, elevates intracellular ROS and sensitises systems to sub-toxic doses of oxidative agents; recovery of cell viability can be achieved through the administration of exogenous glutathione ${ }^{43,44}$. For the first time with MSCs, we used this technique to confirm the findings of the proteomic, bioinformatics and qPCR analysis and in doing so revealed the importance of glutathione to the clonogenic survival of MSCs following exposure to $\mathrm{Ag}^{+}$ions. Our data also highlighted a reduction in colony number during prolonged BSO treatment, although reduction in in vitro cell viability was not observed by other researchers investigating the effects over shorter time periods, with a greater BSO concentration ${ }^{45,46}$. Additionally, the inhibition of the glutathione system potentiated the effect of sub-toxic $\mathrm{Ag}^{+}$concentrations, which overwhelmed the remaining defence mechanisms of the normally sliver-tolerant sub-population.

Oxidative DNA damage resulting from ROS can be induced in cells exposed to $\mathrm{Ag}^{+47}$. While the risk of transversion mutations is abrogated by several DNA repair mechanisms, the upregulation of proteins involved in global genome nucleotide excision repair were identified in our assessment of MSC clonogenicity following silver treatment. This suggests that neutralisation of ROS alone is not sufficient to enable colony formation in sliver-treated MSCs and that other pathways are employed in order to maintain the MSC population.

Increased oxidative stress impairs fracture healing in rodents ${ }^{48,49}$. In a study by Lippross et al. a decline in the quality of bone remodelling was described and has been supported by evidence of reduced osteogenic differentiation of MSCs in vitro while under conditions of oxidative stress, including those caused by $\mathrm{Ag}^{+}$exposure $\mathrm{e}^{4-52}$. Osteoblast activity plays an important role in the formation of the hard callus during secondary fracture healing, an event that occurs several weeks following the initial injury. We determined that the retention time of silver within the bone was minimal in our model, additionally, we found that adipogenesis was differentially altered dependent on the timing of exposure. While a limitation of our study is our measurement of one indicator of osteogenesis (ALP), with further work investigating other markers, for example by qPCR, required to provide more robust information, we report that little effect on the osteogenic capacity of confluent MSCs and cell viability was observed even while under the $10 \mu \mathrm{M} \mathrm{Ag}^{+}$conditions, a concentration that is in line with the maximum silver elution characteristics measured using the rat in vivo model. Of interest, however, was the significant decline in MSC clonogenicity that was discovered while under these same conditions. The importance of MSC number at a fracture site was highlighted by Hernigou et al. and it is conceivable that our observation of reduced clonogenicity during conditions of oxidative stress may explain the reports of reduced healing in fracture models with localised induced $\operatorname{ROS}^{48,49,53}$. Further investigation of MSC function following in vivo exposure to the silver coated implants would have provided valuable additional insight.

Together, our data indicate that clinically relevant silver concentrations remain sub-toxic to MSCs at high density, but can result in a decreased colony-forming capacity. Tolerant CFU-fs show upregulated mechanisms to neutralise ROS and minimise the impact of DNA damage, with the implication that those cells maintain their osteogenic capacity, allowing the progression of fracture healing. These findings indicate that silver may have a role to play in reducing the incidence of fracture fixation related infection, bringing potential benefits to both patients and healthcare system.

\section{Materials and methods}

The following methods describe the investigation of $\mathrm{Ag}^{+}$release from intramedullary implants, translating this to in vitro methods that assessed the effect on MSC function.

Preparation and characterisation of silver coated implants. Titanium alloy (Ti-6Al-4V) rods (20 mm length $\times 1.1 \mathrm{~mm}$ diameter) were passivated in $10 \mathrm{M}$ sodium hydroxide at a temperature of $60^{\circ} \mathrm{C}$ (pins, $1 \mathrm{~h} 50 \mathrm{~min}$ ) followed by incubation in $0.1 \mathrm{M}$ silver nitrate (Alfa Aesar) at $60^{\circ} \mathrm{C}$ for $1 \mathrm{~h}$, to achieve a targeted silver dose of $\sim 50 \mu \mathrm{g} / \mathrm{cm}^{2}$. All implants were gamma sterilised prior to surgery (25-40 kGy).

Total silver analysis was determined pre- and post-implantation (where appropriate). Individual implants were placed in 1:2 (v/v) nitric acid/ $\mathrm{dH}_{2} \mathrm{O}$ and incubated overnight at room temperature (RT). Solutions were vortexed and diluted 1:1000 (v/v) in 1\% nitric acid. Silver content was determined against an $\mathrm{Ag}^{+}$standard $(0.2-20 \mathrm{ppb})$ using the Agilent 8800 ICP-MS Triple Quad (Stockport, UK) in the presence of a 500 ppb rhodamine internal standard.

Extended time-point repeat challenge microbiology testing of silver coated pins. Silver coated pins in cryovial tubes (NUNC, UK) were inoculated with $1 \mathrm{~mL}$ Staphylococcus aureus ATCC $25923\left(1 \times 10^{4} \mathrm{CFU} /\right.$ $\mathrm{mL}$ ) in $10 \%$ Foetal Bovine Serum (FBS)/PBS and incubated horizontally $\left(37^{\circ} \mathrm{C}\right.$ with agitation at $\left.150 \mathrm{rpm}\right)$. Each time point $(24 \mathrm{~h}, 7,14,21$ and 33 days, $\mathrm{n}=5 /$ time point) received a $50 \%$ media change at $3-4$ day intervals, replacing $500 \mu \mathrm{L}$ of test suspension with and equivalent volume of fresh $10 \%$ FBS in PBS (Note: the $24 \mathrm{~h}$ time point received no media change and 33 day sample received no media change between days 18 and 27).

For each time point, samples allotted for analysis were transferred to a fresh cryovial and $1 \mathrm{~mL}$ of fresh inoculum added to each. Following a further $24 \mathrm{~h}$ incubation, the pins were placed in to clean tubes, washed $6 \times$ with sterile PBS before transfer to neutraliser $(0.85 \% \mathrm{NaCl}, 0.4 \%$ sodium thioglycollate, $1 \%$ Tween 20$)$ and the colonising bacteria from the surface were recovered as described in ASTM E2871-13. Briefly this comprised vortex mixing for $30 \mathrm{~s}$, followed by sonication for $30 \mathrm{~s}(45 \mathrm{kHz})$, this was repeated, with a final vortex before serial dilution in MRD and plating on to Petrifilm (3M, UK). All Petrifilm were incubated for at least $48 \mathrm{~h}$ at $32^{\circ} \mathrm{C}$ before counting. Twenty-four hour samples underwent the same recovery process, without the additional $24 \mathrm{~h}$ re-challenge following initial incubation. 
In vitro cell culture. Mesenchymal stem/stromal cells (MSCs) of human origin were isolated from bone marrow aspirate (Lonza) or excised bone donated by patients undergoing primary arthroplasty surgery (NHS, Clifton Park Hospital, UK). Informed consent was obtained from all subjects. Collections and all methods were carried out in accordance with relevant guidelines and regulations under approval from the University of York Biology Ethical Committee Review Board and NHS Local Research Ethics Committee. Clonal MSCs, termed Y201, were generated from primary bone marrow derived MSCs engineered to overexpress human telomerase reverse transcriptase (hTERT) ${ }^{29}$. The use of Y201 MSCs as a model system has been indicated where appropriate. Both primary MSCs and Y201 MSCs were expanded in Dulbecco's modified Eagle's medium (DMEM) supplemented with $10 \% \mathrm{FBS}, 100$ Units/mL Penicillin and $100 \mu \mathrm{g} / \mathrm{mL}$ Streptomycin $\left(37^{\circ} \mathrm{C}, 5 \% \mathrm{CO}_{2}\right)$. Human osteoblasts (hObs) were purchased from PromoCell (Germany) and cultured in osteoblast growth media (PromoCell). Cells were passaged using $0.25 \%$ Trypsin-EDTA once 90\% confluent. All reagents purchased from Sigma (Poole, UK) unless otherwise stated.

In vitro viability and proliferation. Primary MSCs seeded at $3.125 \times 10^{4} \mathrm{cells} / \mathrm{cm}^{2}$ in 96 -well plates were incubated for $24 \mathrm{~h}$ before addition of an $\mathrm{Ag}^{+}$dose response (range 8.75-100 $\mu \mathrm{M}, \mathrm{Ag}_{2} \mathrm{SO}_{4}$, Alfa Aesar, Heysham, UK). After a further $24 \mathrm{~h}$, WST-1 reagent was added and the optical density read at $440 \mathrm{~nm}$ and $600 \mathrm{~nm}$ after $1 \mathrm{~h}$. Results were converted to viability as a percentage of the untreated control. Proliferation over $72 \mathrm{~h}$ was assessed using 5-ethynyl-2'-deoxyuridine (EdU) incorporation (ThermoFisher, Loughborough, UK). MSCs were seeded for $24 \mathrm{~h}$ in to 24 -well plates $\left(2.5 \times 10^{3}\right.$ cells $\left./ \mathrm{cm}^{2}\right)$ before addition of $\mathrm{Ag}^{+}(0-10 \mu \mathrm{M})$. After $72 \mathrm{~h}$, cultures were counterstained with Hoechst and image analysis performed using ImageJ, providing the percentage of EdU positive cells.

CFU-f and CFU-Ob formation. Primary MSC cultures at clonal density $\left(10 \mathrm{cells} / \mathrm{cm}^{2}\right)$ were established in DMEM + 20\% HyClone FBS (GE Healthcare, Little Chalfont, UK) to generate CFU-f (colony-forming unit fibroblast). As for the assessment of viability and proliferation, medium was changed $24 \mathrm{~h}$ after seeding for that containing $\mathrm{Ag}^{+}(0-10 \mu \mathrm{M})$. Medium was changed twice per week, with one subset of plates receiving $\mathrm{Ag}^{+}$for the first 3 days only, after which medium was without silver. Colony formation (defined as $\geq 50$ cells) was assessed after 14 days following crystal violet staining $(0.05 \% \mathrm{w} / \mathrm{v}$ crystal violet) for $20 \mathrm{~min}$.

CFU-Ob (colony-forming unit osteoblast) were generated as described for CFU-f, but supplementing the medium with osteogenic additives at the medium change $24 \mathrm{~h}$ after seeding $\left(50 \mu \mathrm{g} / \mathrm{mL} \mathrm{L}\right.$-ascorbic acid, $10^{-8} \mathrm{M}$ dexamethasone, $3 \mathrm{mM} \beta$-glycerophosphate). Alkaline phosphatase (ALP) positive colonies were identified after 14 days using $1 \%$ Fast Red TR/0.2\% Naphthol AS-MX applied for 2 min.

Differentiation of MSCs. Primary MSCs were culture expanded, seeded in to well-plates for $24 \mathrm{~h}$ prior to the addition of osteogenic differentiation medium (described above) $\pm 10 \mu \mathrm{M} \mathrm{Ag}^{+}$. As before, one group received $\mathrm{Ag}^{+}$free medium after 3 days while maintaining $10 \mu \mathrm{M} \mathrm{Ag}^{+}$in the second treatment group. Adipogenic differentiation media $\left(10^{-6} \mathrm{M}\right.$ Dexamethasone, $500 \mu \mathrm{M}$ 3-Isobutyl-1-methylxanthine, $1 \mu \mathrm{g} / \mathrm{mL}$ Insulin from bovine pancreas, $100 \mu \mathrm{M}$ Indomethacin) and osteogenic media, were changed twice per week with time points of 21 days (adipogenic) and seven and 14 days (osteogenic). Controls underwent the same medium changes (with/without $\left.\mathrm{Ag}^{+}\right)$and time points.

Adipogenic cultures were rinsed (1xPBS) and fixed for ten minutes in 10\% formaldehyde (Polysciences, USA) followed by a rinse with $\mathrm{dH}_{2} \mathrm{O}$ and the addition of Oil Red $\mathrm{O}$ working solution for five minutes. The stain was removed and the wells washed with $60 \%$ Isopropanol before a final rinse with tap water. Lipid bound Oil Red O was quantified through removal with $99 \%$ Isopropanol and the optical density measured at $520 \mathrm{~nm}$ (Multiskan GO, Thermo Fisher, UK,). For osteogenic analysis, at the specified time points, cultures were lysed and ALP activity analysed against a p-nitrophenol standard (pNP, $0-250 \mathrm{nmol} / \mathrm{mL})$ using a pNPP substrate $(5 \mathrm{mM})$. Optical density $(405 \mathrm{~nm})$ was measured after $1 \mathrm{~h}\left(37^{\circ} \mathrm{C}\right)$. Data were normalised to DNA, quantified using PicoGreen (ThermoFisher), measured at Ex: $485 \mathrm{~nm} / \mathrm{Em}$ : $538 \mathrm{~nm}$. Differentiation following MSC expansion as CFU-f was determined, with MSCs seeded at clonal density $\pm 10 \mu \mathrm{M} \mathrm{Ag}{ }^{+}$for 14 days, before differentiation in $\mathrm{Ag}^{+}$free medium. Time points and analysis were as previously described.

CFU-f formation following $\mathrm{Ag}^{+}$exposure. The continued clonogenic capacity of MSCs following $\mathrm{Ag}^{+}$ treatment and the existence of an $\mathrm{Ag}^{+}$tolerant subpopulation that maintained colony formation under further $\mathrm{Ag}^{+}$exposure were assessed using a two-stage CFU-f assay (see Fig. 3a for schematic description). In brief, primary MSCs seeded at clonal density were cultured in medium $\left(0,1\right.$ or $\left.10 \mu \mathrm{M} \mathrm{Ag}^{+}\right)$and assigned as 'Stage 1 '. Medium was changed twice per week for 14 days, after which, cells from the same treatment group (e.g. control) were pooled. MSCs were re-seeded in to further 6-well plates at clonal density ('Stage 2') and medium $\left( \pm \mathrm{Ag}^{+}\right)$ changed as before. After 14 days, the colonies of the 'Stage 2' plates were counted and diameters measured. Assessment of CFU-f diameter was performed using the Zeiss Zen 2.3 Lite software.

CFU-f formation in conditioned medium. The effect of environmental signals on MSC (primary and Y201 MSCs) clonogenicity was determined by culturing clonally seeded MSCs in conditioned medium (MSC$\mathrm{CM}$ ). Tissue culture flasks of MSCs at 70\% confluence were rinsed with phosphate buffered saline (PBS) and further culture in serum free DMEM for $24 \mathrm{~h}$. Medium was removed, centrifuged and filtered $(0.45 \mu \mathrm{m})$ and HyClone FBS added to give $20 \%$ (stored $2-8{ }^{\circ} \mathrm{C}$ ). 'Non-conditioned' (i.e. basal) or 'Conditioned' medium were used during media changes $\left( \pm 10 \mu \mathrm{M} \mathrm{Ag}^{+}\right)$with colonies assessed as before after 14 days. 
qPCR of oxidative stress pathway. RNA was extracted from primary MSC-generated CFU-f $( \pm 10 \mu \mathrm{M}$ $\mathrm{Ag}^{+}$). CFU-f were pelleted and RNA isolated according to RNeasy Mini Kit protocol (Qiagen, Manchester, UK). cDNA synthesis was performed with 500 ng mRNA per sample reaction using RT $^{2}$ First Strand Kit (Qiagen). QPCR was performed using the $\mathrm{RT}^{2}$ Human Oxidative Stress PCR Array (Qiagen, Cat: PAHS-065Z) with SYBR Green detection run on the Thermo Fisher Quantstudio 3 Real-Time PCR System $\left(95^{\circ} \mathrm{C}\right.$ for 10 min followed by 40 cycles of $95^{\circ} \mathrm{C}$ for $15 \mathrm{~s}, 60^{\circ} \mathrm{C}$ for one minute, a final melt curve of $95^{\circ} \mathrm{C}$ for $15 \mathrm{~s}, 60{ }^{\circ} \mathrm{C}$ for one minute, $95^{\circ} \mathrm{C}$ for one second). Fold-changes were calculated for each donor, normalising to $\beta$-2-microglobulin (B2M).

Global proteomic analysis of CFU-f. $\mathrm{Ag}^{+}$cultured Y201 CFU-f were washed and lysed in urea lysis buffer added at 5:1 (v:v) of the cell pellet before undergoing three bursts of microtip sonication at 15 watts, cooling on ice for one minute between bursts. Reduction, alkylation, trypsin digestion and peptide fractionation was performed as described by Geoghegan et al. for Aedes aegypti midguts ${ }^{54}$. Peptide fractions were loaded onto an UltiMate 3000 RSLCnano HPLC system (Thermo) equipped with a PepMap $100 \AA \mathrm{C}_{18}, 5 \mu \mathrm{m}$ trap column $(300 \mu \mathrm{m} \times 5 \mathrm{~mm}$ Thermo $)$ and a PepMap, $2 \mu \mathrm{m}, 100 \AA \mathrm{A}_{18}$ EasyNano nanocapillary column $(75 \mu \mathrm{m} \times 500 \mathrm{~mm}$, Thermo). Flow rate was $300 \mathrm{~nL} / \mathrm{min}$ and the column temperature $40^{\circ} \mathrm{C}$. Separation used gradient elution of aqueous $1 \%(\mathrm{v}: \mathrm{v})$ formic acid (solvent $\mathrm{A}$ ) and aqueous $80 \%(\mathrm{v}: \mathrm{v})$ acetonitrile containing $1 \%(\mathrm{v}: \mathrm{v})$ formic acid (solvent B). The linear multi-step gradient profile was: 3-10\% B over $7 \mathrm{~min}, 10-35 \%$ B over $30 \mathrm{~min}, 35-99 \%$ B over $5 \mathrm{~min}$ and then proceeded to wash with $99 \%$ solvent B for $4 \mathrm{~min}$. The nanoLC system was interfaced with an Orbitrap Fusion hybrid mass spectrometer and positive ESI-MS and MS ${ }^{2}$ spectra were acquired using Xcalibur software (version 4.0, Thermo). $\mathrm{MS}^{1}$ spectra were acquired in the Orbitrap with Easy-IC: 120,000 resolution, scan range: $m / z 375-1500$. Data-dependent acquisition was performed in top speed mode using a $1 \mathrm{~s}$ cycle and 50 s dynamic exclusion. $\mathrm{MS}^{2}$ spectra were acquired in the linear ion trap with HCD activation energy of $32 \%$. Peak area derived relative quantification was obtained from non-conflicting precursor intensities using Progensis QI as detailed previously ${ }^{55}$. Relative abundances were normalised between high $\mathrm{pH} \mathrm{C}_{18}$ fractions using total ion intensity. Database searching was performed against the human subset of the UniProt database (Date 20180123; 20,244 sequences; 11,338,534 residues) using Mascot (Matrix Science Ltd., version 2.5.1), filtering identifications through the Percolator algorithm to achieve a global 1\% FDR. Accepted quantifiable proteins were further filtered to require a minimum of two unique peptides. Multiple test corrected q-values for differential abundance were calculated in Progenesis QI with the Hochberg and Benjamini FDR estimation additionally applied for further stringency.

Immunocytochemistry of CFU-f (GCLM and TRX). Detection of $\gamma$-glutamylcysteine synthetase (GCLM) and thioredoxin (TRX) was in CFU-f of primary MSCs cultured with $\mathrm{Ag}^{+}$on glass coverslips (using a HeLa cell positive control). Slides were fixed with ice cold 100\% methanol (10 min) rinsed with PBS and incubated with $5 \%$ goat serum/PBS at room temperature $(1 \mathrm{~h})$. Primary antibody incubation $(1 \mathrm{~h}, \mathrm{RT})$ also occurred in $5 \%$ goat serum/PBS $10 \mu \mathrm{g} / \mathrm{mL}$ polyclonal rabbit anti-GCLM IgG (Cat: NBP1-33405, RRID: AB_2107841, Novusbio, UK) and $4 \mu \mathrm{g} / \mathrm{mL}$ polyclonal rabbit anti-thioredoxin IgG (Cat: NBP2-48873, RRID AB_2819005, Novusbio). Slides were rinsed before incubation with $10 \mu \mathrm{g} / \mathrm{mL}$ Alex fluor 647 -conjugated goat anti-rabbit IgG $(\mathrm{H}+\mathrm{L})$ cross adsorbed (1 h, RT; Cat: A-21244, RRID: AB_2535812, ThermoFisher) and counterstained with $2 \mu \mathrm{g} / \mathrm{mL}$ DAPI. Coverslips were mounted in aqueous mounting media (Abcam, Cambridge, UK). All immunofluorescence imaging was performed using the Zeiss Axio Imager.M2 LSM 710 confocal microscope (Carl Zeiss, Cambridge, UK).

Inhibition of glutathione synthesis and addition of GSH-MEE. CFU-f from primary MSCs were cultured with $\mathrm{Ag}^{+}(0,1,5$ and $10 \mu \mathrm{M})$ in the presence/absence of a pharmacological inhibitor of $\gamma$-glutamylcysteine synthetase, L-buthionine-sulfoxamine (BSO, 0.01, $0.11 \mu \mathrm{M}$ ). Recovery the glutathione pathway in BSO treated cultures was through media supplementation with $2 \mathrm{mM}$ GSH-MEE. Media were changed twice per week with CFU-f number assessed at 14 days.

In vivo $\mathrm{Ag}^{+}$release from intramedullary implants. Study design was approved by the Smith and Nephew Animal Welfare and Ethical Review Board in compliance with the Animals (Scientific Procedures) Act 1986, taking into consideration the requirements for reduction, replacement and refinement. Twelve male Sprague Dawley rats (250-300 g) were randomised in to four groups consisting of three animals per group. Each group was allocated to a time point of $24,48,72 \mathrm{~h}$ or 28 days. Animals were group housed in temperature controlled rooms at $21 \pm 2{ }^{\circ} \mathrm{C}$ with a relative humidity of $55 \pm 10 \%$ and artificial lighting cycle of $12 \mathrm{~h}$ light/dark. Sub-cutaneous antibiotic prophylaxis (Septrin, $24 \mathrm{mg} / \mathrm{kg}$ ), was provided prior to surgery and twice per day for the initial 2 days of the study. In addition, intra-peritoneal injections of Buprenorphine analgesia $(0.05 \mathrm{mg} / \mathrm{kg})$ and medetomidine sedative $(0.1 \mathrm{mg} / \mathrm{kg})$ were provided. Following surgery, buprenorphine $(0.05 \mathrm{mg} / \mathrm{kg}) \mathrm{was}$ administered every eight hours for a minimum of $48 \mathrm{~h}$. Animals were anaesthetised using an Isoflurane/Oxygen/ Nitrous oxide mixture. Access to the femoral canal was via an entry hole in the inter-condylar notch of the left hind leg. The test article was inserted and the wound closed using absorbable sub-articular sutures. Following surgery, the animals underwent X-rays and were returned to individual housing to recover, after which they were returned to the group. Animals were sacrificed at allocated time points and plasma samples prepared from whole blood collected in heparin sodium salt anticoagulant. The femurs were removed and the implants retrieved.

Silver quantification of plasma and femurs. Plasma samples were diluted 1:25 (v/v) in $1 \%$ nitric acid and the $\mathrm{Ag}^{+}$content determined against a matrix matched standard [0.05-2 ppb, $4 \%$ rat plasma (Innovative 
Research)]. Femurs were heated to $700{ }^{\circ} \mathrm{C}$ for four hours and mixed with a $1: 2(\mathrm{v} / \mathrm{v})$ nitric acid/ $\mathrm{dH}_{2} \mathrm{O}$ solution and left overnight at RT. Samples were diluted 1:200 (v/v) in 1\% nitric acid and $\mathrm{Ag}^{+}$quantified as before (0.2-20 ppb).

Statistical analysis. Data were expressed as the mean \pm SEM. In vitro experiments were performed with a minimum of three primary donors. Data were interrogated as stated in the individual methods using appropriate multiple comparison test. Statistical analysis was performed using GraphPad Prism 7 software (GraphPad Software, La Jolla, CA, USA).

Received: 18 December 2019; Accepted: 27 August 2020

Published online: 03 November 2020

\section{References}

1. Thakore, R. V. et al. Surgical site infection in orthopedic trauma: a case-control study evaluating risk factors and cost. J. Clin. Orthop. Trauma 6, 220-226 (2015).

2. Metsemakers, W. J., Smeets, B., Nijs, S. \& Hoekstra, H. Infection after fracture fixation of the tibia: analysis of healthcare utilization and related costs. Injury 48, 1204-1210 (2017).

3. Trampuz, A. \& Zimmerli, W. Diagnosis and treatment of infections associated with fracture-fixation devices. Injury 37(Suppl 2), S59-66 (2006).

4. Williams, I., Venables, W. A., Lloyd, D., Paul, F. \& Critchley, I. The effects of adherence to silicone surfaces on antibiotic susceptibility in Staphylococcusaureus. Microbiology 143, 2407-2413 (1997).

5. Høiby, N., Bjarnsholt, T., Givskov, M., Molin, S. \& Ciofu, O. Antibiotic resistance of bacterial biofilms. Int. J. Antimicrob. Agents 35, 322-332 (2010).

6. Singh, R., Ray, P., Das, A. \& Sharma, M. Penetration of antibiotics through Staphylococcusaureus and Staphylococcusepidermidis biofilms. J. Antimicrob. Chemother. 65, 1955-1958 (2010).

7. Jesaitis, A. J. et al. Compromised host defense on Pseudomonasaeruginosa biofilms: characterization of neutrophil and biofilm interactions. J. Immunol. 171, 4329-4339 (2003).

8. Kristian, S. A. et al. Biofilm formation induces C3a release and protects Staphylococcus epidermidis from IgG and complement deposition and from neutrophil-dependent killing. J. Infect. Dis. 197, 1028-1035 (2008).

9. Thurlow, L. R. et al. Staphylococcusaureus biofilms prevent macrophage phagocytosis and attenuate inflammation in vivo. J. Immunol. 186, 6585-6596 (2011).

10. Elek, S. D. \& Conen, P. E. The virulence of Staphylococcuspyogenes for man. A study of the problems of wound infection. Br. J. Exp. Pathol. 38, 573-586 (1957).

11. Gristina, A. G., Costerton, J. W. \& McGainty, P. L. J. Bacteria-laden biofilms: a hazard of orthopedic prostheses. Infect. Surg. 3, 655-662 (1984).

12. Fuchs, T., Stange, R., Schmidmaier, G. \& Raschke, M. J. The use of gentamicin-coated nails in the tibia: preliminary results of a prospective study. Arch. Orthop. Trauma Surg. 131, 1419-1425 (2011).

13. Shahverdi, A. R., Fakhimi, A., Shahverdi, H. R. \& Minaian, S. Synthesis and effect of silver nanoparticles on the antibacterial activity of different antibiotics against Staphylococcus aureus and Escherichia coli. Nanomed. Nanotechnol. Biol. Med. 3, 168-171 (2007).

14. Morones-Ramirez, J. R., Winkler, J. A., Spina, C. S. \& Collins, J. J. Silver enhances antibiotic activity against Gram-negative bacteria. Sci. Transl. Med. 5, 1-11 (2013).

15. Kuehl, R. et al. Preventing implant-associated infections by silver coating. Antimicrob. Agents Chemother. 60, 2467-2475 (2016).

16. Feng, Q. L. et al. A mechanistic study of the antibacterial effect of silver ions on Escherichiacoli and Staphylococcusaureus. J. Biomed. Mater. Res. 52, 662-668 (2000).

17. Yamanaka, M., Hara, K. \& Kudo, J. Bactericidal actions of a silver ion solution on Escherichiacoli, studied by energy-filtering transmission electron microscopy and proteomic analysis. Appl. Environ. Microbiol. 71, 7589-7593 (2005).

18. Holt, K. B. \& Bard, A. J. Interaction of silver(I) ions with the respiratory chain of Escherichiacoli: an electrochemical and scanning electrochemical microscopy study of the antimicrobial mechanism of micromolar Ag. Biochemistry 44, 13214-13223 (2005).

19. Jung, W. K. et al. Antibacterial activity and mechanism of action of the silver ion in Staphylococcusaureus and Escherichiacoli. Appl. Environ. Microbiol. 74, 2171-2178 (2008).

20. Anas, A. et al. Sequential interactions of silver-silica nanocomposite $\left(\mathrm{Ag}-\mathrm{SiO}_{2} \mathrm{NC}\right)$ with cell wall, metabolism and genetic stability of Pseudomonasaeruginosa, a multiple antibiotic-resistant bacterium. Lett. Appl. Microbiol. 56, 57-62 (2013).

21. Hardes, J. et al. Reduction of periprosthetic infection with silver-coated megaprostheses in patients with bone sarcoma. J. Surg. Oncol. 101, 389-395 (2010).

22. Wafa, H. et al. Retrospective evaluation of the incidence of early periprosthetic infection with silver-treated endoprostheses in high-risk patients: case-control study. Bone Joint J. 97-B, 252-257 (2015).

23. Donati, F. et al. Silver-coated hip megaprosthesis in oncological limb savage surgery. Biomed Res. Int. https://doi. org/10.1155/2016/9079041 (2016).

24. Bielby, R., Jones, E. \& McGonagle, D. The role of mesenchymal stem cells in maintenance and repair of bone. Injury 38, S26-S32 (2007).

25. Marsell, R. \& Einhorn, T. A. The biology of fracture healing. Injury 42, 551-555 (2011).

26. Kanda, Y., Hinata, T., Kang, S. W. \& Watanabe, Y. Reactive oxygen species mediate adipocyte differentiation in mesenchymal stem cells. Life Sci. 89, 250-258 (2011).

27. Yoshimaru, T., Suzuki, Y., Inoue, T., Niide, O. \& Ra, C. Silver activates mast cells through reactive oxygen species production and a thiol-sensitive store-independent $\mathrm{Ca}^{2+}$ influx. Free Radic. Biol. Med. 40, 1949-1959 (2006).

28. Cortese-Krott, M. M. et al. Silver ions induce oxidative stress and intracellular zinc release in human skin fibroblasts. Free Radic. Biol. Med. 47, 1570-1577 (2009).

29. James, S. et al. Multiparameter analysis of human bone marrow stromal cells identifies distinct immunomodulatory and differentiation-competent subtypes. Stem Cell Rep. 4, 1004-1015 (2015).

30. Jenks, P. J., Laurent, M., McQuarry, S. \& Watkins, R. Clinical and economic burden of surgical site infection (SSI) and predicted financial consequences of elimination of SSI from an English hospital. J. Hosp. Infect. 86, 24-33 (2014).

31. Papakostidis, C. et al. Prevalence of complications of open tibial shaft fractures stratified as per the Gustilo-Anderson classification. Injury 42, 1408-1415 (2011).

32. Vernè, E. et al. Surface characterization of silver-doped bioactive glass. Biomaterials 26, 5111-5119 (2005). 
33. Mohandas, A., Krishnan, A. G., Biswas, R., Menon, D. \& Nair, M. B. Antibacterial and cytocompatible nanotextured Ti surface incorporating silver via single step hydrothermal processing. Mater. Sci. Eng. C 75, 115-124 (2017).

34. Hendricks, K. J. et al. Elution characteristics of tobramycin from polycaprolactone in a rabbit model. Clin. Orthop. Relat. Res. 392, $418-426$ (2001).

35. Pittenger, M. F. Multilineage potential of adult human mesenchymal stem cells. Science 284, 143-147 (1999).

36. Castro-Malaspina, H. et al. Characterization of human bone marrow fibroblast colony-forming cells (CFU-F) and their progeny. Blood 56, 289-301 (1980).

37. Bianco, P., Kuznetsov, S. A., Riminucci, M. \& Gehron Robey, P. Postnatal skeletal stem cells. Methods Enzymol. 419, 117-148 (2006).

38. Hansen, J. M., Zhang, H. \& Jones, D. P. Differential oxidation of thioredoxin-1, thioredoxin-2, and glutathione by metal ions. Free Radic. Biol. Med. 40, 138-145 (2006).

39. Cuypers, A. et al. Cadmium stress: an oxidative challenge. Biometals 23, 927-940 (2010).

40. Shukla, G. S. et al. Cadmium-mediated oxidative stress in alveolar epithelial cells induces the expression of $\gamma$-glutamylcysteine synthetase catalytic subunit and glutathione S-transferase $\alpha$ and $\pi$ isoforms: Potential role of activator protein-1. Cell Biol. Toxicol. 16, 347-362 (2000).

41. Chen, D.-J. et al. Proteomic analysis of secreted proteins by human bronchial epithelial cells in response to cadmium toxicity. Proteomics 15, 3075-3086 (2015).

42. Dekkers, S. et al. Multi-omics approaches confirm metal ions mediate the main toxicological pathways of metal-bearing nanoparticles in lung epithelial A549 cells. Environ. Sci. Nano 5, 1506-1517 (2018).

43. Wellner, V. P., Anderson, M. E., Puri, R. N., Jensen, G. L. \& Meister, A. Radioprotection by glutathione ester: transport of glutathione ester into human lymphoid cells and fibroblasts. PNAS 81, 4732-4735 (1984).

44. Lu, H. et al. Chemotherapy triggers HIF-1-dependent glutathione synthesis and copper chelation that induces the breast cancer stem cell phenotype. PNAS 112, E4600-E4609 (2015).

45. Ito, K. et al. Reactive oxygen species act through p38 MAPK to limit the lifespan of hematopoietic stem cells. Nat. Med. 12, 446-451 (2006).

46. Arai, Y., Miyayama, T. \& Hirano, S. Difference in the toxicity mechanism between ion and nanoparticle forms of silver in the mouse lung and in macrophages. Toxicology 328, 84-92 (2015).

47. Jiang, X. et al. Multi-platform genotoxicity analysis of silver nanoparticles in the model cell line CHO-K1. Toxicol. Lett. 222, 55-63 (2013).

48. Göktürk, E. et al. Oxygen-free radicals impair fracture healing in rats. Acta Orthop. 66, 473-475 (1995).

49. Lippross, S. et al. Nrf2 deficiency impairs fracture healing in mice. Calcif. Tissue Int. 95, 349-361 (2014).

50. Cortizo, M. C., de Mele, M. F. L. \& Cortizo, A. M. Metallic dental material biocompatibility in osteoblastlike cells: correlation with metal ion release. Biol. Trace Elem. Res. 100, 151-168 (2004).

51. Kim, W.-K., Meliton, V., Bourquard, N., Hahn, T. J. \& Parhami, F. Hedgehog signaling and osteogenic differentiation in multipotent bone marrow stromal cells are inhibited by oxidative stress. J. Cell. Biochem. 111, 1199-1209 (2010).

52. Sengstock, C., Diendorf, J., Epple, M., Schildhauer, T. A. \& Köller, M. Effect of silver nanoparticles on human mesenchymal stem cell differentiation. Beilstein J. Nanotechnol. 5, 2058-2069 (2014).

53. Hernigou, P. H., Poignard, A., Beaujean, F. \& Rouard, H. Percutaneous autologous bone-marrow grafting for nonunions. Influence of the number and concentration of progenitor cells. J. Bone Jt. Surg. 87, 1430-1437 (2005).

54. Geoghegan, V. et al. Perturbed cholesterol and vesicular trafficking associated with dengue blocking in Wolbachia-infected Aedesaegypti cells. Nat. Commun. 8, 526 (2017).

55. Dowle, A. A., Wilson, J. \& Thomas, J. R. Comparing the diagnostic classification accuracy of iTRAQ, peak-area, spectral-counting, and emPAI methods for relative quantification in expression proteomics. J. Proteome Res. 15, 3550-3562 (2016).

\section{Acknowledgements}

We are grateful to staff and patients of Clifton Park Hospital for samples.

\section{Author contributions}

P.S. performed all cell biology and associated data acquisition, analysis and figure preparation, while also being involved with all experimental and study planning, figure and manuscript preparation. J.V., K.B. and J.C. were responsible for the acquisition and analysis of microbiology, microCT and ICP-MS; A.D. performed and analysed the proteomics. JV (Fig. 1a), KB (Fig. 1c) and AD (Fig. 4b, Figs S1 and S3) also prepared the relevant figures and text for the manuscript. JD was responsible for completion of the in vivo study, while $\mathrm{MH}$ prepared implants and technology development. DW, AH and PG were involved in experimental design and planning the work. $\mathrm{AH}$ and $\mathrm{PG}$ had overall supervision responsibility, PG also contributed to manuscript preparation and writing.

\section{Funding}

The York Centre of Excellence in Mass Spectrometry was created thanks to a major capital investment through Science City York, supported by Yorkshire Forward with funds from the Northern Way Initiative, and subsequent support from EPSRC (EP/K039660/1; EP/M028127/1). Financial support was provided by Smith \& Nephew plc, Innovate UK (55657-420144) and the Tissue Engineering and Regenerative Therapies Centre Versus Arthritis (21156).

\section{Competing interests}

P Souter's PhD was funded by Smith and Nephew plc. Paul Souter, John Vaughan, Kerry Butcher, Jim Cunningham, James Dodd, Michael Hall, Darren Wilson and Alan Horner are current or were previous employees of Smith and Nephew plc. Dr Wilson has a patent application concerning the silver technology use in the in vivo study. Adam Dowle and Paul Genever declare no potential conflict of interest.

\section{Additional information}

Supplementary information is available for this paper at https://doi.org/10.1038/s41598-020-76087-1.

Correspondence and requests for materials should be addressed to P.G.

Reprints and permissions information is available at www.nature.com/reprints. 
Publisher's note Springer Nature remains neutral with regard to jurisdictional claims in published maps and institutional affiliations.

(c) (i) Open Access This article is licensed under a Creative Commons Attribution 4.0 International License, which permits use, sharing, adaptation, distribution and reproduction in any medium or format, as long as you give appropriate credit to the original author(s) and the source, provide a link to the Creative Commons licence, and indicate if changes were made. The images or other third party material in this article are included in the article's Creative Commons licence, unless indicated otherwise in a credit line to the material. If material is not included in the article's Creative Commons licence and your intended use is not permitted by statutory regulation or exceeds the permitted use, you will need to obtain permission directly from the copyright holder. To view a copy of this licence, visit http://creativecommons.org/licenses/by/4.0/.

(c) The Author(s) 2020 